\title{
Comparative Chondrogenesis of Human Cells in a 3D Integrated Experimental/Computational Mechanobiology Model
}

Manuela Teresa Raimondi, ${ }^{1,2}$ Elisa Bonacina, ${ }^{2}$ Gabriele Candiani, ${ }^{1}$ Matteo Laganà,${ }^{2}$ Elena Rolando, ${ }^{1}$ Giuseppe Talò, ${ }^{2}$ Daniele Pezzoli, ${ }^{1}$ Roberto D'Anchise, ${ }^{2}$ Riccardo Pietrabissa, ${ }^{1}$ Matteo Moretti ${ }^{2}$ Corresponding Author: manuela.raimondi@polimi.it

${ }^{1}$ Politecnico di Milano, Milano, Italy, and ${ }^{2}$ IRCCS Galeazzi Orthopaedic Institute, Milano, Italy

\section{Introduction}

In articular cartilage in vitro regeneration, flow combined to cyclic pressurization is believed to be particularly effective in maintaining cell viability and in up regulating the biosynthesis of phenotypic matrix proteins [1]. To investigate cartilage regeneration from ostheoarthritic (OA) human cells, we developed a model of cartilage regeneration in which cell pellets are cultured in a bioreactor system, under combined regimens of perfusion and hydrodynamic pressurization.
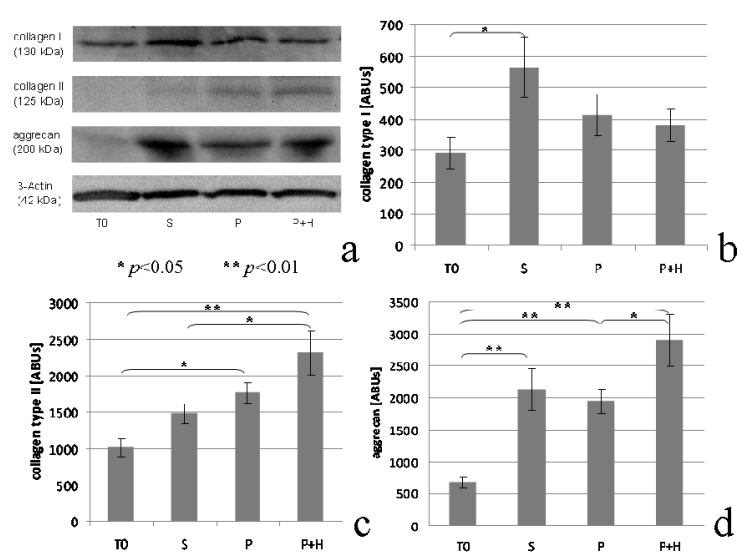

Fig. 1. Western Blots (a) for the pellets content in collagen type I (b), collagen type II (c) and $\operatorname{aggrecan}(\mathrm{d})$.

\section{Materials and Methods}

Chondrocytes were isolated from human knee cartilage harvested from 4 arthroplasty patients. Cells were expanded for two passages, trypsinized and cell pellets were obtained.

The pellets were divided into 4 groups: group $\mathrm{T} 0$, at four days of static culture, and groups $\mathrm{S}$, $\mathrm{P}$, and $\mathrm{P}+\mathrm{H}$ at further 7 days of static, perfused and perfused+pressurized culture, respectively. The analyses performed on the pellets were: DNA content, metabolic activity, sGAG content, collagen type I, II and aggrecan content by Western Blotting, and standard histology. Oneway ANOVA for non-correlated samples and pair-wise Tukey HSD tests were performed.
The oxygen concentration and fluid shears were calculated, both in the pellet and in the culture medium, in the static vs perfused conditions. The computations were performed with the finite element code Comsol Multiphysics.

\section{Results}

Synthesis of collagen type II and aggrecan was highest in pellets perfused and pressurized (Fig $1)$, in which the lowest oxygen concentration was around $40 \%$ saturation (Fig 2). Synthesis of collagen type I was highest in statically cultured pellets, in which oxygen concentration dropped at a hypoxic $1 \%$ saturation value and shear stress was absent.

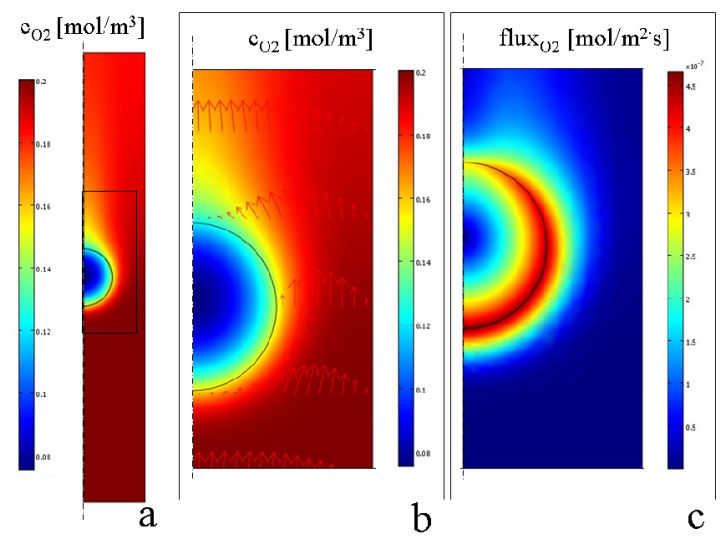

Fig. 2. Multi-physics simulation showing oxygen concentration (a-b) and diffusive flux (c) in the bioreactor-cultured pellets.

\section{Discussion and Conclusions}

We demonstrated that human OA chondrocytes synthesize phenotypic matrix proteins in response to combined regimens of flow and pressurization in a pellet model. This implies that these cells can be an adequate source in a de novo regeneration context, provided that the culture conditions are optimized.

\section{References}

1. Moretti M.(2008) Biom Mat Eng 18,273-8.

Disclosures Authors have nothing to disclose. 